\title{
The Use of Allogeneic Leukocytes Infusion in Cancer Immunotherapy
}

\author{
Yishu Tang ${ }^{1 *}$, Chunxia Zhou ${ }^{2}$, Wenbo Ma², Dongmei Wang ${ }^{2}$, Shuren Zhang ${ }^{2}$ \\ ${ }^{1}$ Department of Laboratory Medicine, The First Affiliated Hospital of Chongqing Medical University, Chongqing, \\ People's Republic of China \\ ${ }^{2}$ Department of Immunology, Cancer Institute, Peking Union Medical College,Chinese Academy of Medical \\ Sciences, Beijing, China
}

*Corresponding Author: Yishu Tang, Department of Laboratory Medicine, The First Affiliated Hospital of Chongqing Medical University, No. 1 Youyi Road, Yuzhong District, Chongqing, 400016, China, Tel: +86 23 89012735; Fax: +86 23 89012735; E-mail: tangyishu111@,163.com

Received: 16 Jan 2018; Accepted: 28 May 2018; Published: 30 May 2018

\begin{abstract}
Mixed allogeneic lymphocyte response has activated considering $\mathrm{T}$ lymphocytes and elicit type 1 cytokines which induced antitumor immunity. Using TC-1 cancer model, we demonstrated the response of inactivated allogeneic leukocytes infusion (ALI) to generate antitumor immune response. In TC-1 tumor model, ALI induced a delayed cancer development and a prolonged survival. Our study illustrated that ALI might elicit strong antitumor immune response. The method strategy would be easy and efficient strategy in tumor immunotherapy.
\end{abstract}

Keywords: Allogeneic Leukocytes; Cancer Immunotherapy

\section{Introduction}

Allogeneic transplantation was being used as a successful method for tumor therapy. Under the clear illustration of transplantation immunology, it is realized that alloantigen was an immunostimulator to elicit an immune effect in host vs. graft (HVG) or graft vs. host (GVH) 1]. In the GVH and HVG biology, there were similar mechanisms to antitumor effects, including cytolysis, antigen-presenting cells (APCs) revitalite, and IFN- $\gamma$ secreting type T cells. So theses mechanisms might be used in the tumor therapy. 
Graft rejection represents an immune effect which was persistent and intensity. In the allogeneic transplantation, the remarkable host $\mathrm{T}$ cell effect to allogeneic major histocompatibility complex (MHC) molecules. A recent study demonstrated the usage for the allogeneic reaction to induce the host weak T cell effect to caner Antogens [2]. This theoretical perspective develops an attractive immunotherapy method in the people with cancer. The response of graft rejection to elicit antitumor effect was illustrated in a clinical test of skin transplantation from HLA unmatched donors 3,4]. In the report, we illustrated the therapeutic potentiality of inactivation MHC unmatched allogeneic leukocytes to prevent GVHD. The prevention of GVHD made the donor cells to have a much higher disparity of MHC with host cells. Our study showed antineoplastic capacity elicited by MHC-unmatched allogeneic leukocytes transplantation.

\section{Materials and methods}

\subsection{Mice and cell lines}

Inbred female C57BL/6 (B6, H-2) and BALB/c mice (8-10 weeks) were purchased from the Experimental Animal Institute of Peking Union Medical College. All protocols were in accordance with recommendations for the proper care of laboratory animals.

TC-1 tumor cells derived from C57BL/6 mice co-transformed with HPV-16 E6, E7 and c-Haras oncogenes were provided by Dr. T.C. Wu from Johns Hopkins University.

\subsection{Cell vaccine preparation}

Murine splenocytes were separated from freshly spleens of BALB/c mice. For inactivating splenocytes, $25 \mathrm{mg} / \mathrm{mL}$ mitomycin C (MMC) were taken into the cell suspension in a concentration of $1 \times 10^{7}$ cells $/ \mathrm{mL}$. And the cells were incubated for $60 \mathrm{~min}$. At last the cell were washed with phosphate-buffered saline (PBS) and resuspended.

\subsection{Cancer therapeutic protocol}

On Day 0, mice were injected with 1 X $10^{5}$ TC-1 tumor cells subcutaneously (s.c.). On Day 10, when the tumors grew approximately $50 \mathrm{~mm}^{3}$, the mice were divided into 3 groups as follows: the PBS control group; ALI groups $\left(4 \times 10^{8}, 2 \times 10^{8}\right)$; On Day 11, the MMC-inactivated allogeneic leukocytes were administered intratumorally (i.t.) and repeated twice every 3 days. Tumors were monitored every 3 days, and the death of mice was registered. Tumor dimensions were recorded with calipers, and the record were inserted into the following formula: tumor volume $\left(\mathrm{mm}^{3}\right)=0.52 \times\left(\right.$ length $\times$ width $\left.^{2}\right)$.

\subsection{Statistical analysis}

Student's t-test was used to compare means between the 2 groups. Logrank test were analyzed in the survival data. Differences were considered to be significant when $\mathrm{p}<0.05$. Statistical analysis was performed using commercially available software (SPSS 11.0). 


\section{Results}

In the report, we applied the TC- 1 cell as tumor model to show the antitumor influence of ALI. TC-1 cancer from mice were treated with $2 \times 10^{8}, 4 \times 10^{8} \mathrm{MMC}$-inactivated BALB/c leukocytes/mouse. The average sizes of tumors on Day 21 were $844.92 \pm 201.56 \mathrm{~mm}^{3}$ in the $4 \times 10^{8}$ ALI group, whereas those given PBS injections developed to an average volume of $2061.573 \pm 397.83 \mathrm{~mm}^{3}$ (Fig $\left.1 \mathrm{~A}, \mathrm{P}<0.05\right)$.

As shown in Figure 1B and Table 1, compared with PBS group, $4 \times 10^{8}$ ALI might extend the lifespan of tumor bearing mice (44.56 vs 31.83$)$.

A

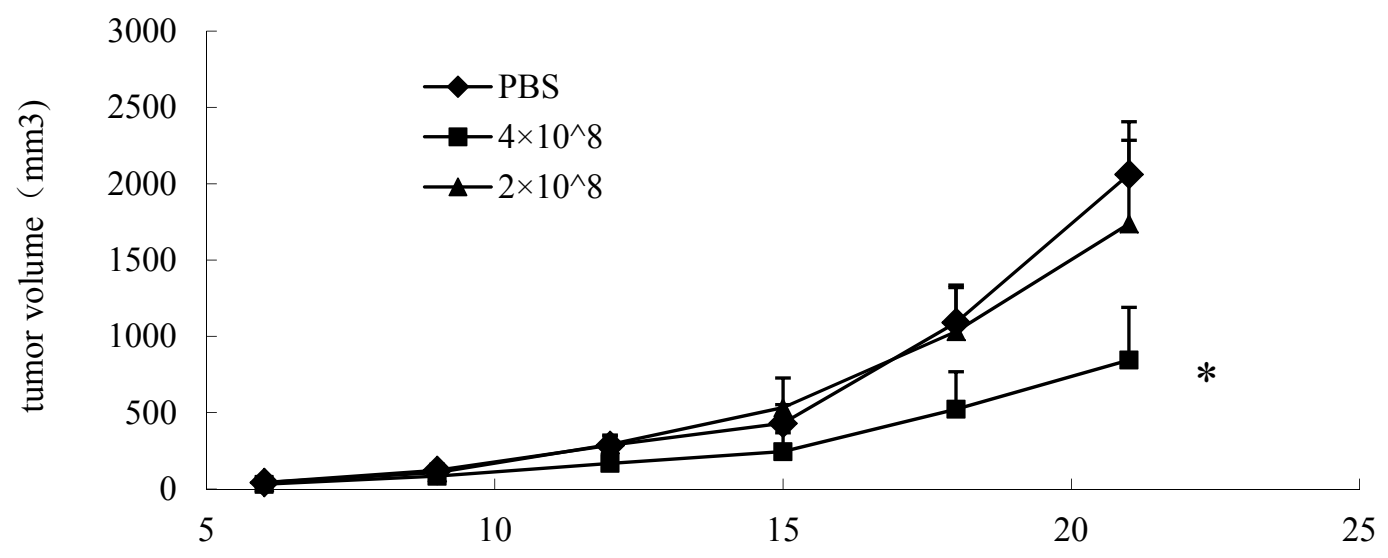

Days after tumor inoculation

B

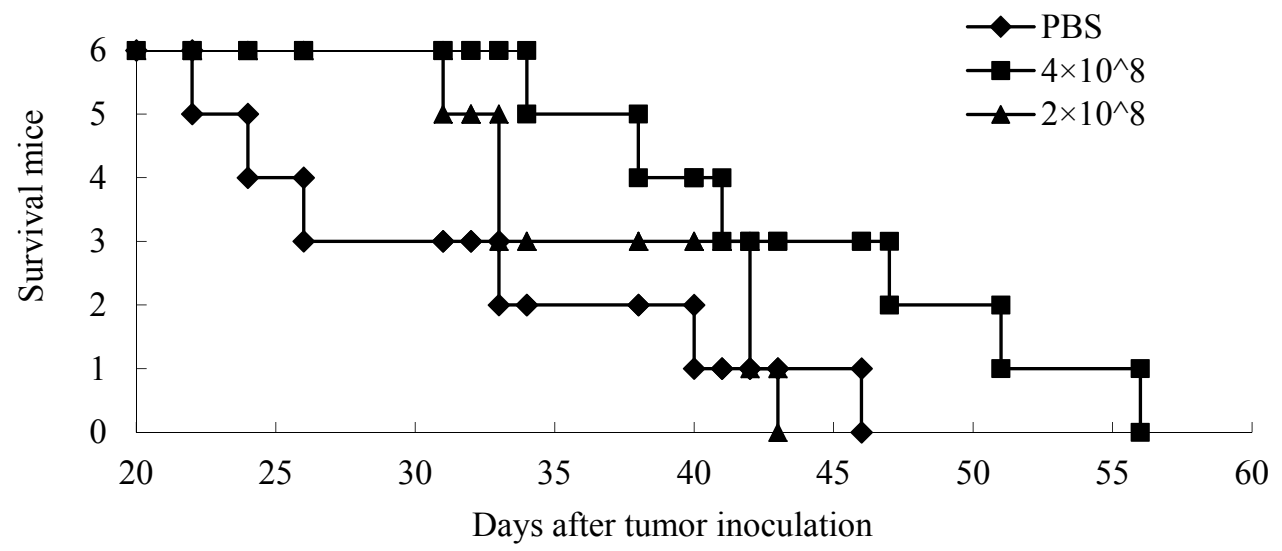

Figure 1: The antitumor effects of ALI. C57BL/6 mice were inoculated s.c. with 1 x105 TC-1 cells. When the tumors reached an average diameter of about $5 \mathrm{~mm}$, the mice were randomized and treated with ALI or PBS on Day 10. The same treatments were repeated on Day 13 and Day 16. (A) Tumor sizes of B6 mice treated with 2 or $4 \times 108$ inactivated BALB/c leukocytes infusion. The results are demonstrated as mean $\pm \mathrm{SD}$. ${ }^{*} \mathrm{p}<0.05$ compared with PBS group on Day 21. (B) Survival of mice per treatment group. N= 6 mice/group. 


\begin{tabular}{|l|l|}
\hline Groups & Median survival(day) \\
\hline PBS & 31.83 \\
\hline $4 \times 10^{8}$ ALI & 44.56 \\
\hline $2 \times 10^{8}$ ALI & 37.33 \\
\hline
\end{tabular}

Table 1: The median survival of mice in tumor immunotherapy

\section{Discussion}

The antitumor response of allogeneic leukocytes was illustrated in previous study [5,6]. All the previous studies were depends on the graft-versus-tumor or graft-versus-leukemia effect which mediated by competent donor cells [7]. In our study, we illustrated that an antitumor immunity could been elicited by an inactivated MHC mismatched allogeneic leukocytes.

ALI might elicit antitumor immunity in 3 methos. First, the anergic and tumor-specific $\mathrm{T}$ cells might be elicited in the polyclonal allogeneic response. Second, the CD4+T-cell direct allorecognition response to MHC-II molecules could afford T-cell help for the self-restricted reaction to cancer peptides. Finally, the inherent immune system might induce an immunostimulatory environment $[8,9]$.

The reports demonstrated that allogeneic response between HLA-unmatched people elicit a markedly raise of type 1 cytokines such as IFN- $\gamma .10$ IFN- $\gamma$ was a key factor $\mathrm{p}$ in promoting host inherent and acquired immune reaction. Other studies have also showed that allogeneic response induce maturation and migration of DCs [10].

The another novel aspect of our study was related to a reversal of lymphopenia in a cancer bearing host. Our results showed that inactivated ALI could inhibit the cancer growth by inducing type- 1 cytokine storm. These cytokines could induce both nonspecific and specific antitumor immune effect. In conclusion, it is sensible to desire the therapeutic modality might be an attractive method in cancer therapy.

\section{Acknowledgements}

This work was supported by a grant from National Key R\&D Program of China (2017YFC0909900), National Natural Science Foundation of China (No 81501818 of Yishu Tang) and the National Key Clinical Specialties Construction Program of China.

\section{Conflict of interest}

The authors declare no conflicts of interest. 


\section{References}

1. Bregni M, Bernardi M, Ciceri F, Peccatori J. Allogeneic stem cell transplantation for the treatment of advanced solid tumors. Springer Semin Immunopathol 26 (2004): 95-108.

2. Fabre JW. The allogeneic response and tumor immunity. Nat Med 7 (2001): 649-652.

3. Muir G, Rajbabu K, Callen C, Fabre JW. Preliminary evidence that the allogeneic response might trigger antitumour immunity in patients with advanced prostate cancer. BJU Int 98 (2006): 989-995

4. Slavin S, Nagler A, Naparstek E, Kapelushnik Y, Aker M, et al. Nonmyeloablative stem cell transplantation and cell therapy as an alternative to conventional bone marrow transplantation with lethal cytoreduction for the treatment of malignant and nonmalignant hematologic diseases. Blood 91 (1998): 756-763.

5. Horowitz MM, Gale RP, Sondel PM, Goldman JM, Kersey J, et al. Graft-versus leukemia reactions after bone marrow transplantation. Blood 75 (1990): 555-562.

6. Ji YH, Weiss L, Zeira M, Abdul-Hai A, Reich S, et al. Allogeneic cell-mediated immunotherapy of leukemia with immune donor lymphocytes to upregulate antitumor effects and downregulate antihost responses. Bone Marrow Transplant 32 (2003): 495-504.

7. Assudani DP, Horton RB, Mathieu MG, McArdle SE, Rees RC. The role of CD41 T cell help in cancer immunity and the formulation of novel cancer vaccines. Cancer Immunol Immunother 56 (2007): 70-80.

8. Matzinger P. Tolerance, danger, and the extended family. Annu Rev Immunol 12 (1994): 991-1045.

9. Medzhitov R, Janeway C, Jr. Innate immunity. N Engl J Med 343 (2000): 338-344.

10. Laurin D, Kanitakis J, Bienvenu J, Bardin C, Bernaud J, et al. Allogeneic reaction induces dendritic cell maturation through proinflammatory cytokine secretion. Transplantation 77 (2004): 267-275.

Citation: Yishu Tang, Chunxia Zhou, Wenbo Ma, Dongmei Wang, Shuren Zhang. The Use of Allogeneic Leukocytes Infusion in Cancer Immunotherapy. Archives of Clinical and Biomedical Research 2 (2018): 151155 . 